\title{
Synthesis and Antidepressant Activity of Some New 5-(1H-Indol-3-yl)-3-(substituted aryl)-4,5-dihydroisoxazoline Derivatives
}

\author{
Pravin O. Patil and Sanjay B. Bari \\ Department of Pharmaceutical Chemistry, R. C. Patel Institute of Pharmaceutical Education and Research, Shirpur, Maharashtra, \\ Dhule 425405, India
}

Correspondence should be addressed to Pravin O. Patil; rxpatilpravin@yahoo.co.in

Received 20 June 2012; Revised 28 July 2012; Accepted 13 August 2012

Academic Editor: Volker Boehm

Copyright (C) 2013 P. O. Patil and S. B. Bari. This is an open access article distributed under the Creative Commons Attribution License, which permits unrestricted use, distribution, and reproduction in any medium, provided the original work is properly cited.

\begin{abstract}
The present study refers to the synthesis of new antidepressant candidates using the indole scaffold. In an attempt to identify potential lead antidepressant agents, a number of indole molecules, incorporating isoxazoline, were synthesized by microwaveassisted synthesis. The antidepressant activity of the synthesized compounds (3a-3n) was evaluated by forced swim test in mice and their locomotor activity was assessed using actophotometry. The present paper showed significant antidepressant activity for all compounds of the series and no significant change in locomotor activity of mice. Compounds $\mathbf{3} \mathbf{d}$ and $\mathbf{3} \mathbf{j}$ were found to be potent molecules of this series, when compared with the reference drugs imipramine and fluoxetine. It clearly demonstrated that replacement of aromatic core by appropriate heterocycles such as pyridine and pyrrole on the 5-(1H-Indol-3-yl)-3-(Phenyl)-4,5dihydroisoxazoline (3a) would generate more potent derivatives. Thus, these compounds can serve as potential leads for further antidepressant studies.
\end{abstract}

\section{Introduction}

Depression is a prevalent psychiatric disorder with estimates reaching as high as $21 \%$ of the world population. The World Health Organization (WHO) predicts that it will be the second leading cause of death by the year 2020 due to complications arising from stress and the cardiovascular system [1]. Although a broad range of antidepressants are available today, a significant proportion of these patients will not respond to treatment or will show only partial response [2]. Clinically used antidepressants have several limitations and side effects which demand continuous development of novel, efficient, and safe drugs for the treatment of depression.

In an attempt to synthesize and evaluate new compounds as antidepressant, we report, herein, synthesis and biological evaluation of a number of indole derivatives bearing isoxazolines. The indole framework is a medicinally relevant scaffold and has become widely identified as a privileged structure or pharmacophore due to its different pharmacological actions [3-5].
Literature survey also suggests the potential of the indolyl isoxazoline derivatives as anti-inflammatory [6] and antimicrobial agents $[7,8]$. Compounds containing the isoxazole nucleus have been reported to possess diverse pharmacological activities [9-12]. The indole moiety is present in approved as well as experimental drugs [13-16] and the isoxazoline moiety in experimental drugs $[17,18]$.

Amongst the various methods available for the synthesis of isoxazolines, Tiwari et al. reported synthesis of quinoline isoxazolines under ultrasound irradiation [19]. Panda et al. reported synthesis of indolyl isoxazole [20], whereas Kidwai et al. reported the first solid-phase regioselective synthesis of isoxazole [21]. The microwave-assisted synthesis was found advantageous over conventional reactions [22, 23].

Therefore, in the present investigation, it was envisaged that if these two pharmacophores are linked together this would generate new molecular templates (Figure 1), which are likely to exhibit antidepressant-like action in animal models. 


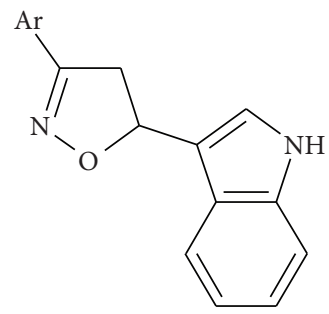

Ar: substituted aryl, heteroaryl, and naphthyl

FIGURE 1: General structure of proposed scaffold as antidepressant agents.

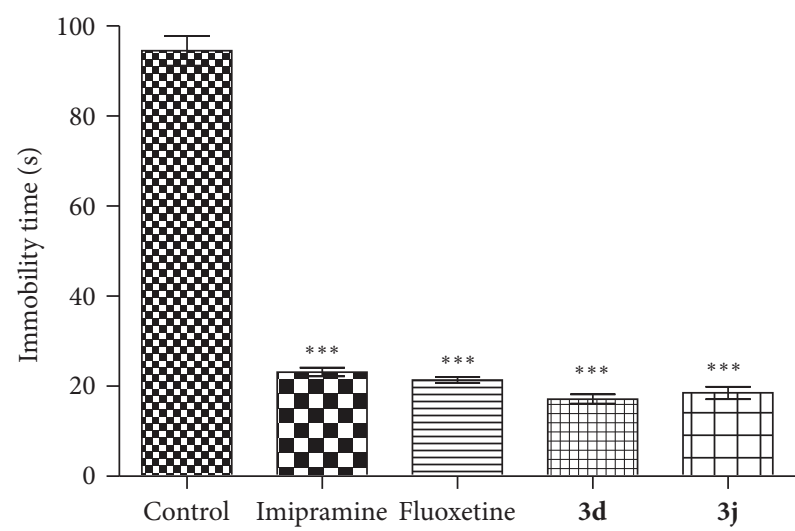

Figure 2: Effect of treatment with $\mathbf{3 d}$ and $\mathbf{3 j}$ ( $20 \mathrm{mg} / \mathrm{kg}$, i.p.), imipramine $(20 \mathrm{mg} / \mathrm{kg}$, i.p.), and fluoxetine $(20 \mathrm{mg} / \mathrm{kg}$, i.p.) on the immobility time in forced swim test. (Values are represented as mean \pm S.E.M. $(n=6)$. Values are significant at ${ }^{* * *} P<0.001$, compared with control group.)

\section{Results and Discussion}

2.1. Chemistry. In the present work, the title compounds, $(\mathbf{3} \mathbf{a}-\mathbf{3 n})$, were synthesized by utilizing the reaction sequence shown in Scheme 1. Thus, indole (1a) reacted with DMF in the presence of phosphorus oxychloride $\left(\mathrm{POCl}_{3}\right)$ to produce 3-formylindole (1b) [24], which further reacted with substituted aryl ketones using piperidine as a base in ethylene glycol under microwave irradiation conditions to yield (2a-2n) [25]. The reaction of equimolar quantities of 2a-2n with hydroxylamine hydrochloride under microwave irradiation resulted in the formation of the target derivatives 3a-3n (Table 1). The structures of the final compounds were confirmed by ${ }^{1} \mathrm{H}$ NMR, ${ }^{13} \mathrm{C}$ NMR, IR, mass spectrometry, and elemental (C, H, N) analysis.

The IR spectra of the compounds $\mathbf{3 a} \mathbf{- 3 n}$ revealed absorption bands in the region of $1630-1550 \mathrm{~cm}^{-1}$ corresponding to $\mathrm{C}=\mathrm{N}$ stretching. Absence of carbonyl $(\mathrm{C}=\mathrm{O})$ peak around $1710-1650 \mathrm{~cm}^{-1}$ reveals the formation of indolyl isoxazolines. In the ${ }^{1} \mathrm{H}$ NMR spectrum, $\mathrm{C} 4\left(\mathrm{H}_{a}, \mathrm{H}_{b}\right)$ and $\mathrm{C} 5\left(\mathrm{H}_{x}\right)$ protons of isoxazolines ring resonated as a pair of doublets at $3.50-3.70(\delta \mathrm{ppm}), 3.70-3.85(\delta \mathrm{ppm})$, and $5.50-5.65$ $(\delta \mathrm{ppm})$. These splittings are owing to vicinal coupling with the two magnetically nonequivalent $\mathrm{C} 4$ methylene protons of the ring.
2.2. Antidepressant Activity. The compounds $\mathbf{3 a}-\mathbf{3 n}$ were evaluated for antidepressant activity by forced swim test (FST) in mice at dose of $100 \mathrm{mg} / \mathrm{kg}$ and compared with the standard drugs imipramine $(20 \mathrm{mg} / \mathrm{kg})$ and fluoxetine $(20 \mathrm{mg} / \mathrm{kg})$. There were no mortality and noticeable behavioural changes in acute oral toxicity for all the groups tested. The synthesized compounds were found to be safe upto $2000 \mathrm{mg} / \mathrm{kg}$ body weight. Initially, dose-dependent study of compound $3 \mathbf{a}$ at different doses $(25,50,100$, and $200 \mathrm{mg} / \mathrm{kg}$, i.p.) were performed to ensure the maximum effective dose for new synthesized compounds as antidepressant in FST. From this study, we found that $100 \mathrm{mg} / \mathrm{kg}$ is the maximum effective dose and therefore was selected for further pilot study of antidepressant-like effects of compounds 3b-3n in FST. Antidepressant activity was assessed as mean immobility time in seconds, and data has been presented as mean \pm S.E.M as shown in (Table 2).

Results of FST revealed that all compounds exhibited significant antidepressant activity as becomes evident from their decrease in duration of immobility (DID) values compared to the control group and high \%DID values. Compounds $\mathbf{3 d}$ and $3 \mathbf{j}$ were found to be the most potent derivatives from the series, at the same time, $\mathbf{3} \mathbf{a}$ and $\mathbf{3} \mathbf{f}$ showed moderate activity. Further, in order to compare the antidepressant effect of active compounds $\mathbf{3} \mathbf{d}$ and $\mathbf{3} \mathbf{j}$ with that of the standard drugs, a separate study was carried out by employing doses of $20 \mathrm{mg} / \mathrm{kg}$ i.p. of $\mathbf{3 d}, \mathbf{3 j}$, imipramine and fluoxetine; the results are presented in Figure 2.

Compounds $\mathbf{3 d}$ and $\mathbf{3 j}$ at the dose of $20 \mathrm{mg} / \mathrm{kg}$ i.p exhibited significant antidepressant activity compared with imipramine and fluoxetine. The preliminary SAR of indolyl isoxazolines suggested that replacement of the phenyl ring by the heterocycles pyridine, pyrrole, and so forth. led to an increase of antidepressant activity (3d, 3e, and $3 \mathbf{j})$. Compounds bearing electron-withdrawing groups (F, Br, and $\mathrm{Cl}$ ) on the para position of the aromatic core showed good antidepressant activity $(\mathbf{3 c}, \mathbf{3 h}, \mathbf{3 m}, \mathbf{3 n})$, whereas electronreleasing groups $\left(\mathrm{CH}_{3}, \mathrm{OH}\right.$, and $\left.\mathrm{OCH}_{3}\right)$ at para as well as ortho position of the aromatic nucleus caused decrease in antidepressant activity $(\mathbf{3 b}, 3 \mathbf{g}, 3 \mathbf{i}, 3 \mathbf{k}, 3 \mathbf{3})$. Therefore, such compounds would represent a useful matrix for the development of a new class of clinically useful antidepressant agents and deserve further investigation and derivatization.

2.3. Locomotor Activity. The antidepressant activities of isoxazolines seem not to be associated with any motor effects, since they did not show significant $(P<0.05)$ change in locomotor function of mice as compared to vehicle-treated group (Table 2) indicating that the new compounds have no excitatory or inhibitory action on central nervous system, which eliminated the probability of false-positive results in forced swimming test.

\section{Conclusion}

In summary, we developed a practical, fast, ecofriendly, and cost effective procedure for the synthesis of new $5-(1 \mathrm{H}$ Indol-3-yl)-3-(4-substituted aryl)-4,5-dihydroisoxazoline derivatives under microwave irradiation. The remarkable 


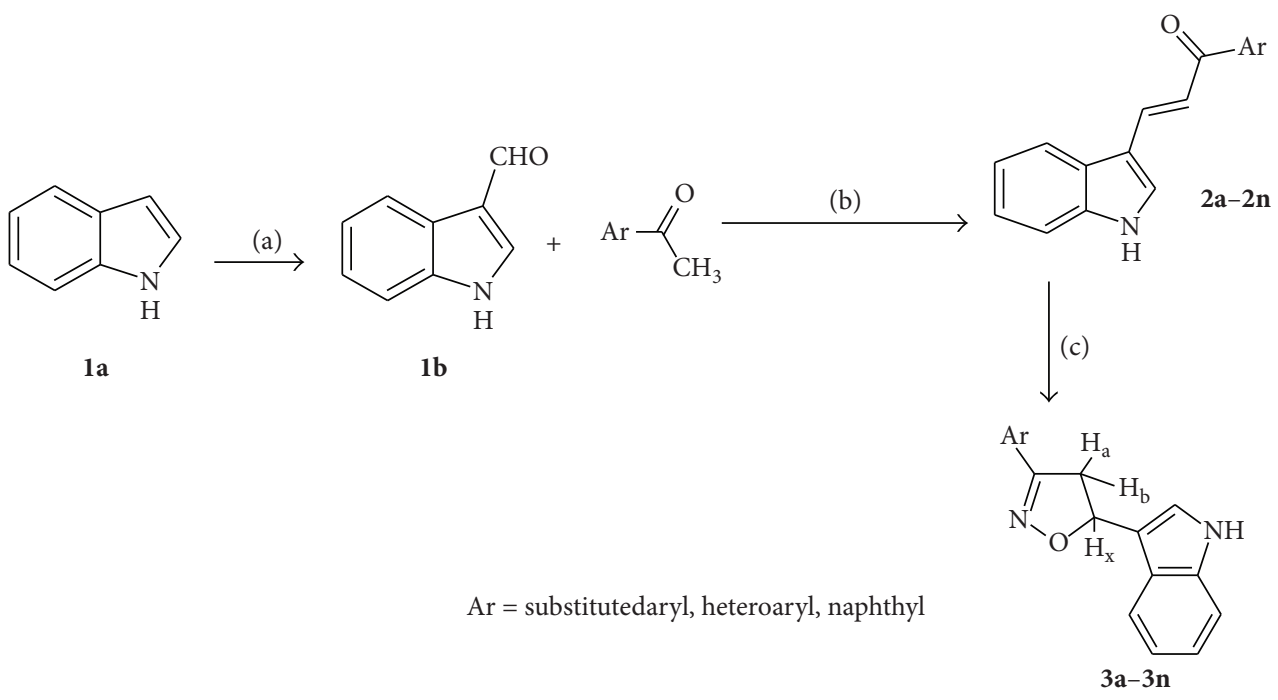

Scheme 1: Synthetic route for the synthesis of target compounds (3a-3n). Reagents and conditions: (a) DMF, $\mathrm{POCl}_{3}$; (b) piperidine, ethylene glycol, mw, $420 \mathrm{~W}, 10 \mathrm{~min}$, target temperature $100^{\circ} \mathrm{C}$; (c) hydroxylamine hydrochloride, $\mathrm{EtOH}, \mathrm{AcOH}, \mathrm{mw}, 455 \mathrm{~W}, 35 \mathrm{~min}$, target temperature $95-98^{\circ} \mathrm{C}$

advantages offered by microwave-assisted reactions over the conventional syntheses are the reduced reaction times, high purity, and better yields of the products. All synthesized compounds were evaluated for antidepressant activity in FST. Among all the derivatives tested in the present study, compounds $\mathbf{3 d}$ and $\mathbf{3} \mathbf{j}$ exhibited promising antidepressant activity comparable to that of the imipramine and fluoxetine. It was clearly demonstrated that the introduction of the appropriate heterocyclic ring on the basic scaffold led to potent derivatives. Hence, the present compounds can act as potential leads for further antidepressant studies.

\section{Experimental}

4.1. General Remarks. All solvents and reagents were used as obtained from commercial sources without further purification. Melting points were recorded and are uncorrected on an Elico Melting Point Apparatus using open capillary tube. All microwave-assisted reactions were performed using a scientific microwave oven (catalyst electromagnetic system) with a power of $800 \mathrm{~W}$ specially designed for organic synthesis. Thin-layer chromatography (TLC) was performed on precoated plates $\mathrm{SiO}_{2}$ (silica gel 60, F 254, Merck) to monitor the progress of the reaction and purity of the compounds, spots being visualized under iodine vapors or UV-light. Silica gel (60-120 mesh size, Merck) was used for column chromatography. All NMR spectra were recorded on a Bruker Avance III, $400 \mathrm{MHz}$ spectrometer with $\mathrm{CDCl}_{3} / \mathrm{DMSO}$ as solvent using tetramethyl silane (TMS) as internal reference standard; chemical shifts are reported in ppm $(\delta)$. IR spectra of compounds were recorded on a Shimadzu IR 48 spectrophotometer with DRS technology. Mass spectra were recorded on a 410 Prostar Binary LC with 500 MS IT PDA detectors with direct infusion mass with ESI and APCI Negative and Positive mode ionization. Elemental analyses were performed on a Vario-EL III CHNOS elemental analyzer.

General Procedure for Synthesis of 5-(1H-Indol-3-yl)-3(substituted aryl)-4,5-dihydroisoxazoline Derivatives (3a-3n). To a mixture of 1-(1H-indol-3-yl)-3-(substituted aryl)-prop1-en-3-ones (1 mmoL) and hydroxylamine hydrochloride ( $1 \mathrm{mmol}$ ) in $20 \mathrm{~mL}$ EtOH was added a catalytic amount of acetic acid in $100 \mathrm{~mL}$ round bottom flask and subjected to microwave irradiation at 455 watt for specified time to attain the set temperature of $95-98^{\circ} \mathrm{C}$. Most of the chalcone was consumed at this time (monitored by TLC); the reaction mixture was cooled to room temperature and was poured into crushed ice. The precipitate was separated by filtration and washed with water to get the desired products, which were purified by column chromatography (Chloroform/Acetone 18:1 as eluant). Physical data and percentage yields of all the synthesized compounds are represented in (Table 1 ).

5-(1H-Indol-3-yl)-3-(phenyl)-4,5-dihydroisoxazoline

(3a). Yield: $87.64 \%$, yellow solid; reaction time: 36 minutes; m.p.: 161-164 C; IR (KBr): cm $3315(\mathrm{~N}-\mathrm{H}), 1620$ (C=C), 1548 $(\mathrm{C}=\mathrm{N}) ;{ }^{1} \mathrm{H} \mathrm{NMR}\left(400 \mathrm{MHz} \mathrm{CDCl}_{3}, \mathrm{ppm}\right) 6.02-6.07$ (dd, $1 \mathrm{H}), 3.55-3.62(\mathrm{dd}, 1 \mathrm{H}), 3.69-3.70(\mathrm{dd}, 1 \mathrm{H}), 7.09-7.76(\mathrm{~m}$, $10 \mathrm{H}), 8.175$ (brs, $1 \mathrm{H}) .{ }^{13} \mathrm{C} \mathrm{NMR}\left(100 \mathrm{MHz} \mathrm{CDCl}_{3}, \mathrm{ppm}\right): 41$, 73.0, 156.6, 130.02, 128.8, 129.9, 126.7, 111.4, 125.2, 136.9, $111.5,120.5,119.4,120.2,122.6$, MS: $\mathrm{m} / z$ with +ve mode: $263.2\left(\mathrm{M}^{+\cdot}\right), 264.1(\mathrm{M}+1)$. Anal. Calcd. for $\mathrm{C}_{17} \mathrm{H}_{14} \mathrm{~N}_{2} \mathrm{O}: \mathrm{C}$, 77.84; H, 5.38; N, 10.68. Found: C, 77.71; H, 5.58; N, 10.59\%.

5-(1H-Indol-3-yl)-3-(4-methylphenyl)-4,5-dihydroisoxazoline (3b). Yield: 81.71\%; yellow solid; reaction time: 37 minutes; m.p.: 167-168 ${ }^{\circ}$; IR (KBr): $\mathrm{cm}^{-1} 3408(\mathrm{~N}-\mathrm{H}), 1608$ (C=C), $1630(\mathrm{C}=\mathrm{N})$; ${ }^{1} \mathrm{H} \mathrm{NMR}\left(400 \mathrm{MHz} \mathrm{CDCl}_{3}, \mathrm{ppm}\right) 2.39$ (s, 3H), 5.99-6.04 (dd, 1H), 3.53-3.60 (dd, 1H), 3.68-3.74 (dd, 1H), 
TABLE 1: Data of 5-(1H-Indol-3-yl)-3-(substituted aryl)-4,5-dihydroisoxazoline derivatives 3a-3n produced via Scheme 1.

\begin{tabular}{|c|c|c|c|}
\hline Compound & $\mathrm{Ar}$ & Yield $(\%)^{\mathrm{a}}$ & m.p. $\left({ }^{\circ} \mathrm{C}\right)$ [lit. m.p.] \\
\hline $3 \mathbf{a}$ & & 87.6 & $61-164$ \\
\hline $3 b$ & & 81.7 & $167-168(167)[26]$ \\
\hline $3 c$ & & 84.3 & $178-179$ \\
\hline $3 d$ & & 74.1 & 198-199 \\
\hline $3 e$ & & 71.9 & $201-203$ \\
\hline $3 f$ & & 81.2 & $206-208$ \\
\hline $3 g$ & & 76.4 & $171-176$ \\
\hline $3 \mathrm{~h}$ & & 74.6 & $207-208$ \\
\hline $3 \mathbf{i}$ & & 70.6 & $164-167$ \\
\hline $3 \mathbf{j}$ & & 75.1 & $183-184$ \\
\hline $3 k$ & & 78.9 & $174-176$ \\
\hline 31 & & 77.3 & $181-183$ \\
\hline $3 \mathrm{~m}$ & & 72.9 & $191-193$ \\
\hline $3 n$ & & 80.6 & $194-197$ \\
\hline
\end{tabular}

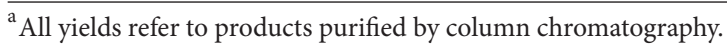

7.09-7.64 (m, 9H), 8.15 (brs, $1 \mathrm{H}) .{ }^{13} \mathrm{CNMR}(100 \mathrm{MHz}$ $\left.\mathrm{CDCl}_{3}, \mathrm{ppm}\right): 19.4,39.0,71.2,154.5,127.4,124.6,125,138.2$, $117.2,123.1,134.8,109.5,120.5,113.4,118.0,127.4, \mathrm{MS}: \mathrm{m} / z$ with +ve mode: $277.2\left(\mathrm{M}^{+*}\right), 278.1(\mathrm{M}+1)$. Anal. Calcd. for $\mathrm{C}_{18} \mathrm{H}_{16} \mathrm{~N}_{2} \mathrm{O}$ : C, 78.24; H, 5.84; N, 10.14. Found: C, 78.17; H, $5.93 ; \mathrm{N}, 9.38 \%$.

5-(1H-Indol-3-yl)-3-(4-chlorophenyl)-4,5-dihydroisoxazoline (3c). Yield: $93.98 \%$, yellow solid; reaction time: 34 minutes; m.p.: $178-179^{\circ} \mathrm{C}$; IR (KBr): $\mathrm{cm}^{-1} 3417(\mathrm{~N}-\mathrm{H}), 1618(\mathrm{C}=\mathrm{C})$, $1591(\mathrm{C}=\mathrm{N}) ;{ }^{1} \mathrm{H}$ NMR (400 MHz $\left.\mathrm{CDCl}_{3}, \mathrm{ppm}\right) 6.03-6.08$ (dd, 1H), 3.27-3.59 (dd, 1H), 3.52-3.59 (dd, 1H), 7.10-7.68 (m, 9H), 8.15 (brs, $1 \mathrm{H}) .{ }^{13} \mathrm{C} \mathrm{NMR}\left(100 \mathrm{MHz} \mathrm{CDCl}_{3}, \mathrm{ppm}\right.$ ): 40.8, 77.9, 156.2, 128.4, 127.9, 129, 136.9, 115.3, 125, 135.97, $111.5,122.5,119.3,115.5,126.7$, MS: $\mathrm{m} / z$ with +ve mode: $297.2\left(\mathrm{M}^{+\cdot}\right), 298.1(\mathrm{M}+1)$. Anal. Calcd. for $\mathrm{C}_{17} \mathrm{H}_{13} \mathrm{ClN}_{2} \mathrm{O}: \mathrm{C}$, 68.81; H, 4.42; N, 9.44. Found: C, 68.53; H, 4.32; N, 9.40\%.

5-(1H-Indol-3-yl)-3-(2-pyridyl)-4,5-dihydroisoxazoline (3d). Yield: $74.12 \%$, yellow solid; reaction time: 41 minutes; m.p.: 198-199 ${ }^{\circ}$; IR (KBr): $\mathrm{cm}^{-1} 3205(\mathrm{~N}-\mathrm{H}), 1598$ (C=C), 1622 $(\mathrm{C}=\mathrm{N}) ;{ }^{1} \mathrm{H}$ NMR (400 MHz, DMSO, ppm) 6.01-6.98 (dd, $1 \mathrm{H}), 3.63-3.69$ (dd, $1 \mathrm{H}), 3.79-3.87(\mathrm{dd}, 1 \mathrm{H}), 6.98-8.94(\mathrm{~m}$, 9H), 11.17 (brs, $1 \mathrm{H}) .{ }^{13} \mathrm{C} \mathrm{NMR}$ (100 MHz DMSO, ppm): 40.7, 
TABLE 2: Effect of 5-(1H-Indol-3-yl)-3-(substituted aryl)-4,5-dihydroisoxazoline series of compounds in forced swim test and locomotor activity.

\begin{tabular}{|c|c|c|c|c|}
\hline Sr. no. & Compounds & $\begin{array}{c}\text { Duration of immobility } \\
(\text { sec. }) \\
(\text { mean } \pm \text { SEM })\end{array}$ & $\begin{array}{c}\text { Percentage decrease in } \\
\text { immobility duration } \\
\text { (\% DID) }\end{array}$ & $\begin{array}{c}\text { Locomotor activity scores } \\
\text { for } 10 \text { minutes (sec.) } \\
(\text { mean } \pm \text { SEM })\end{array}$ \\
\hline 1 & $3 a$ & $35.7 \pm 4.3^{* * *}$ & 62.2 & $470 \pm 16.0^{\mathrm{ns}}$ \\
\hline 2 & $3 \mathbf{b}$ & $56.3 \pm 2.0^{* * *}$ & 40.4 & $490 \pm 9.0^{\mathrm{ns}}$ \\
\hline 3 & $3 c$ & $26.2 \pm 2.8^{* * *}$ & 72.3 & $480 \pm 17.0^{\mathrm{ns}}$ \\
\hline 4 & $3 d$ & $11.3 \pm 0.5^{* * *}$ & 88.0 & $480 \pm 13.0^{\mathrm{ns}}$ \\
\hline 5 & $3 e$ & $23.7 \pm 1.1^{* * *}$ & 75.0 & $460 \pm 13.0^{\mathrm{ns}}$ \\
\hline 6 & $3 f$ & $40.2 \pm 4.1^{* * *}$ & 57.5 & $450 \pm 14.0^{\mathrm{ns}}$ \\
\hline 7 & $3 g$ & $50.7 \pm 4.2^{* * *}$ & 46.4 & $430 \pm 29.0^{\mathrm{ns}}$ \\
\hline 8 & $3 h$ & $27.5 \pm 0.9^{* * *}$ & 70.9 & $410 \pm 28.0^{\mathrm{ns}}$ \\
\hline 9 & $3 \mathbf{i}$ & $68.5 \pm 4.6^{* * *}$ & 27.5 & $470 \pm 18.0^{\mathrm{ns}}$ \\
\hline 10 & $3 \mathbf{j}$ & $12.5 \pm 0.9^{* * *}$ & 86.8 & $470 \pm 8.4^{\mathrm{ns}}$ \\
\hline 11 & $3 k$ & $72.5 \pm 4.5^{* * *}$ & 23.3 & $450 \pm 17.0^{\mathrm{ns}}$ \\
\hline 12 & 31 & $54.3 \pm 4.4^{* * *}$ & 42.5 & $460 \pm 17.0^{\mathrm{ns}}$ \\
\hline 13 & $3 m$ & $33.2 \pm 5.7^{* * *}$ & 64.9 & $410 \pm 26.0^{\mathrm{ns}}$ \\
\hline 14 & $3 n$ & $29.3 \pm 2.1^{* * *}$ & 69.0 & $460 \pm 17.0^{\mathrm{ns}}$ \\
\hline 15 & Vehicle & $94.5 \pm 3.3$ & 0.0 & $510 \pm 15$ \\
\hline 16 & Imipramine $(20 \mathrm{mg} / \mathrm{kg})$ & $23.2 \pm 0.9^{* * *}$ & 75.5 & $490 \pm 14^{\mathrm{ns}}$ \\
\hline 17 & Fluoxetine $(20 \mathrm{mg} / \mathrm{kg})$ & $21.3 \pm 0.6^{* * *}$ & 77.4 & $470 \pm 11^{\mathrm{ns}}$ \\
\hline
\end{tabular}

a Data analyzed by one-way ANOVA followed by Dunnett's test. $n=6$; dose $=100 \mathrm{mg} / \mathrm{kg}$. Values are represented as mean \pm S.E.M. Values are significant at ${ }^{* * *} P$ $<0.001$, compared with control group. ns: not significant $(P<0.05)$ as compared to vehicle-treated group.

$77.5,154.7,123.9,147.5,136.8,125.8,150.7,113.4,125.3$ 133.7, $111.8,121.5,119,118.8,124.2$, MS: $m / z$ with +ve mode: 265.14 $\left(\mathrm{M}^{+\cdot}\right)$, Anal. Calcd. for $\mathrm{C}_{16} \mathrm{H}_{13} \mathrm{~N}_{3} \mathrm{O}$ : C, 72.99; $\mathrm{H}, 4.98$; N, 15.96. Found: C, 72.91; H, 5.02; N, 15.79\%.

5-(1H-Indol-3-yl)-3-(2-thienyl)-4,5-dihydroisoxazoline (3e). Yield: $71.88 \%$, yellow solid; reaction time: 37 minutes; m.p.:194-197 C; IR (KBr): $\mathrm{cm}^{-1} 3217$ (N-H), 1623 (C=C), 1568(C=N); ${ }^{1} \mathrm{HNMR}\left(400 \mathrm{MHz} \mathrm{CDCl}_{3}, \mathrm{ppm}\right) 6.03-6.06$ (dd, 1H), 3.52-3.59 (dd, 1H), 3.67-3.74 (dd, 1H), 6.08-7.68 (m, 9H), 8.150 (brs, 1H). $\left.{ }^{13} \mathrm{C} \mathrm{NMR} \mathrm{(100} \mathrm{MHz} \mathrm{CDCl}_{3}, \mathrm{ppm}\right)$ : $40.6,78,155.5,128.4,127.9,129,136.9,115.3,125.1,136$, $111.6,122.7,122.4,119.2,115.3$, MS: $m / z$ with +ve mode: $308.1\left(\mathrm{M}^{+\cdot}\right)$, Anal. Calcd. for $\mathrm{C}_{17} \mathrm{H}_{13} \mathrm{~N}_{3} \mathrm{O}_{3}$ : C, 66.44; H, 4.26; N, 13.67. Found: C, 66.47; H, 4.41; N, 13.54\%.

5-(1H-Indol-3-yl)-3-(2-naphthyl)-4,5-dihydroisoxazoline (3f). Yield: $81.24 \%$, light yellow solid; reaction time: 34 minutes; m.p.: 206-208 ${ }^{\circ}$; IR (KBr): $\mathrm{cm}^{-1} 3253(\mathrm{~N}-\mathrm{H}), 1618(\mathrm{C}=\mathrm{C})$, $1582(\mathrm{C}=\mathrm{N}) ;{ }^{1} \mathrm{H} \mathrm{NMR}\left(400 \mathrm{MHz} \mathrm{CDCl}_{3}, \mathrm{ppm}\right) 6.02-6.07$ (dd, 1H), 3.55-3.62 (dd, 1H), 3.68-3.71 (dd, 1H), 7.02-7.78 (m, 12H), 8.17 (brs, 1H). $\left.{ }^{13} \mathrm{C} \mathrm{NMR} \mathrm{(100} \mathrm{MHz} \mathrm{CDCl}_{3}, \mathrm{ppm}\right)$ : $41,73,156.6,130,128.8,129.9,126.7,111.4,125.1,136.9$, $111.5,120.5,119.3,120.1,122.6$, MS: $m / z$ with +ve mode: 313.21 ( $\left.\mathrm{M}^{+\cdot}\right)$, Anal. Calcd. for $\mathrm{C}_{21} \mathrm{H}_{16} \mathrm{~N}_{2} \mathrm{O}$ : C, 80.75; H, 5.16; N, 8.97. Found: C, 80.62; H, 5.04; N, 9.01\%.

5-(1H-Indol-3-yl)-3-(4-methoxyphenyl)-4,5-dihydroisoxazoline (3g). Yield: $84.57 \%$, yellow solid; reaction time: 40 minutes; m.p.: $171-174^{\circ} \mathrm{C}$; IR (KBr): $\mathrm{cm}^{-1} 3258(\mathrm{~N}-\mathrm{H}), 1602(\mathrm{C}=\mathrm{C})$,
$1612(\mathrm{C}=\mathrm{N}) ;{ }^{1} \mathrm{H}$ NMR (400 MHz $\left.\mathrm{CDCl}_{3}, \mathrm{ppm}\right) 3.79$ (s, 3H), 5.92-6.01 (dd, $1 \mathrm{H}), 3.59-3.61(\mathrm{dd}, 1 \mathrm{H}), 3.63-3.78(\mathrm{dd}, 1 \mathrm{H})$, 7.11-7.61 (m, 9H), 8.16 (brs, $1 \mathrm{H}) .{ }^{13} \mathrm{CNMR}(100 \mathrm{MHz}$ $\left.\mathrm{CDCl}_{3}, \mathrm{ppm}\right): 39,55.6,71.3,153.6,127.4,124.75,124.9$, 138.1, 117.7, 123.1, 134.8, 109.2, 120.1, 113, 118.4, 127.1, MS: $m / z$ with +ve mode: $293.31\left(\mathrm{M}^{+\cdot}\right)$, Anal. Calcd. for $\mathrm{C}_{18} \mathrm{H}_{16} \mathrm{~N}_{2} \mathrm{O}_{2}$ : C, 73.95; H, 5.52; N, 9.58. Found: C, 73.91; H, 5.37; N, 9.46\%.

5-(1H-Indol-3-yl)-3-(4-fluorophenyl)-4,5-dihydroisoxazoline (3h). Yield: $74.62 \%$, yellow solid; reaction time: 34 minutes; m.p.: 207-208 ${ }^{\circ}$; IR (KBr): $\mathrm{cm}^{-1} 3256(\mathrm{~N}-\mathrm{H}), 1608$ (C=C), $1583(\mathrm{C}=\mathrm{N}) ;{ }^{1} \mathrm{HNMR}\left(400 \mathrm{MHz} \mathrm{CDCl}_{3}, \mathrm{ppm}\right)$ 6.03-6.05 (dd, 1H), 3.53-3.59 (dd, 1H), 3.67-3.74 (dd, 1H), 6.09-7.67 (m, 9H), 8.154 (brs, 1H). ${ }^{13} \mathrm{CNMR}\left(100 \mathrm{MHz} \mathrm{CDCl}_{3}, \mathrm{ppm}\right)$ : $40.5,77.8,156.1,128.5,127.9,129.1,136.8,115.3,125.1$, $135.9,111.7,122.5,122.8,120.5,119.3, \mathrm{MS}: m / z$ with +ve mode: $297.32\left(\mathrm{M}^{+\cdot}\right)$, Anal. Calcd. for $\mathrm{C}_{18} \mathrm{H}_{17} \mathrm{FN}_{2} \mathrm{O}$ : C, 72.85; H, 4.67; N, 9.99. Found: C, 72.93; H, 4.41; N, 9.78\%.

5-(1H-Indol-3-yl)-3-(2-hydroxyphenyl)-4,5-dihydroisoxazoline (3i). Yield: $70.62 \%$, light yellow solid; reaction time: 36 minutes; m.p.: $164-167^{\circ} \mathrm{C}$; IR (KBr): $\mathrm{cm}^{-1} 3272(\mathrm{~N}-\mathrm{H}), 3336$ $(\mathrm{OH}), 1618(\mathrm{C}=\mathrm{C}), 1591(\mathrm{C}=\mathrm{N}) ;{ }^{1} \mathrm{H} \mathrm{NMR}\left(400 \mathrm{MHz} \mathrm{CDCl}_{3}\right.$, ppm) 11.81 (s, 1H), 6.03-6.08 (dd, 1H), 3.53-3.59 (dd, $1 \mathrm{H}), 3.61-3.74$ (dd, 1H), 6.07-7.67 (m, 9H), 8.14 (brs, $1 \mathrm{H})$. ${ }^{13}$ C NMR: 41.2, 77.8, 153.7, 127.3, 127.5, 128, 135.8, 115.4, $124.2,135.8,111,122,122.1,119.4,115.5$, MS: $m / z$ with +ve mode: $279.17\left(\mathrm{M}^{+\cdot}\right)$, Anal. Calcd. for $\mathrm{C}_{17} \mathrm{H}_{14} \mathrm{~N}_{2} \mathrm{O}_{2}$ : C, 73.37; H, 5.07; N, 10.07. Found: C, 73.19; H, 5.21; N, 10.29\%. 
5-(1H-Indol-3-yl)-3-(2-pyrolyl)-4,5-dihydroisoxazoline (3j). Yield: $75.12 \%$, yellow solid; reaction time: 37 minutes; m.p.: 183-184 ${ }^{\circ}$; IR (KBr): $\mathrm{cm}^{-1} 3278$ (N-H), 1618 (C=C), 1589 $(\mathrm{C}=\mathrm{N}) ;{ }^{1} \mathrm{H} \mathrm{NMR}\left(400 \mathrm{MHz} \mathrm{CDCl}_{3}, \mathrm{ppm}\right) 13.06(\mathrm{~s}, 1 \mathrm{H})$, 5.97-6.06 (dd, 1H), 3.51-3.62 (dd, $1 \mathrm{H}), 3.66-3.76(\mathrm{dd}, 1 \mathrm{H})$, 7.09-7.67 (m, 8H), 8.14 (brs, 1H). ${ }^{13} \mathrm{CNMR}: 40.7,77.4$, $154.6,123,147.2,136.6,125.7,151.3,113.6,133.5,111.5$, $121.2,119.7,118.3,123.2$, MS: $m / z$ with +ve mode: 252.1 $\left(\mathrm{M}^{+\cdot}\right)$, Anal. Calcd. for $\mathrm{C}_{15} \mathrm{H}_{13} \mathrm{~N}_{3} \mathrm{O}: \mathrm{C}, 71.70 ; \mathrm{H}, 5.21 ; \mathrm{N}$, 16.72. Found: C, 71.57; H, 5.46; N, 16.84\%.

5-(1H-Indol-3-yl)-3-(4-hydroxyphenyl)-4,5-dihydroisoxazoline (3k). Yield: 75.92\%, yellow solid; reaction time: 34 minutes; m.p.:174-176 C; IR (KBr): $\mathrm{cm}^{-1} 3248(\mathrm{~N}-\mathrm{H}), 3317(\mathrm{OH})$, $1622(\mathrm{C}=\mathrm{C}), 1579(\mathrm{C}=\mathrm{N}) ;{ }^{1} \mathrm{HNMR}\left(400 \mathrm{MHz} \mathrm{CDCl}_{3}\right.$, ppm) $11.83(\mathrm{~s}, 1 \mathrm{H}), 6.04-6.08(\mathrm{dd}, 1 \mathrm{H}), 3.51-3.57$ (dd, $1 \mathrm{H}), 3.69-3.71$ (dd, 1H), 6.09-7.64 (m, 9H), 8.15 (brs, 1H).

${ }^{13}$ C NMR: $40.5,77.9,154.5,127.3,127.9,129,135.4,115.6$, $125.4,136.9,111.51,122,122.4,118.2,115.7, \mathrm{MS}: \mathrm{m} / z$ with +ve mode: $279.21\left(\mathrm{M}^{+\cdot}\right)$, Anal. Calcd. for $\mathrm{C}_{17} \mathrm{H}_{14} \mathrm{~N}_{2} \mathrm{O}_{2}$ : C, 73.37; H, 5.07; N, 10.07. Found: C, 73.27; H, 5.18; N, 10.34\%.

5-(1H-Indol-3-yl)-3-(2-methylphenyl)-4,5-dihydroisoxazoline (31). Yield: 77.32\%; light yellow solid; reaction time: 37 minutes; m.p.: $167-168^{\circ} \mathrm{C}$; IR (KBr): $\mathrm{cm}^{-1} 3408(\mathrm{~N}-\mathrm{H}), 1608$ $(\mathrm{C}=\mathrm{C}), 1630(\mathrm{C}=\mathrm{N}) ;{ }^{1} \mathrm{H} \mathrm{NMR}\left(400 \mathrm{MHz} \mathrm{CDCl}_{3}, \mathrm{ppm}\right) 2.39$ (s, 3H), 5.99-6.04 (dd, 1H), 3.53-3.60 (dd, 1H), 3.68-3.74 (dd, 1H), 7.09-7.64 (m, 9H), 8.15 (brs, 1H). ${ }^{13} \mathrm{CNMR}$ (100 MHz $\left.\mathrm{CDCl}_{3}, \mathrm{ppm}\right): 19.4,39,71.2,154.5,127.4,124.6$, 125, 138.2, 117.8, 123.1, 134.8, 109.5, 120.5, 113.4, 118, 127.4, MS: $m / z$ with +ve mode: $277.2\left(\mathrm{M}^{+\cdot}\right), 278.1(\mathrm{M}+1)$. Anal. Calcd. for $\mathrm{C}_{18} \mathrm{H}_{16} \mathrm{~N}_{2} \mathrm{O}$ : C, 78.24; $\mathrm{H}, 5.84 ; \mathrm{N}, 10.14$. Found: $\mathrm{C}$, 78.17; H, 5.93; N, 9.38\%.

5-(1H-Indol-3-yl)-3-(4-bromophenyl)-4,5-dihydroisoxazoline (3m). Yield: $77.32 \%$, yellow solid; reaction time: 36 minutes; m.p.: 191-193 ${ }^{\circ}$; IR (KBr): $\mathrm{cm}^{-1} 3317(\mathrm{~N}-\mathrm{H}), 1614(\mathrm{C}=\mathrm{C})$, $1576(\mathrm{C}=\mathrm{N}) ;{ }^{1} \mathrm{HNMR}\left(400 \mathrm{MHz} \mathrm{CDCl}_{3}, \mathrm{ppm}\right) 6.03-6.06$ (dd, 1H), 3.53-3.58 (dd, 1H), 3.68-3.73 (dd, 1H), 6.08-7.68 (m, 9H), 8.157 (brs, 1H). ${ }^{13} \mathrm{C} \mathrm{NMR}$ (100 $\left.\mathrm{MHz} \mathrm{CDCl}_{3}, \mathrm{ppm}\right)$ : $40.5,77.9,155.5,128.6,127.9,129,136.9,115.3,125.2,136$, $111.6,122.6,122.7,120.2,119.3$, MS: $m / z$ with +ve mode: 342.14 $\left(\mathrm{M}^{+\cdot}\right)$, Anal. Calcd. for $\mathrm{C}_{18} \mathrm{H}_{17} \mathrm{BrN}_{2} \mathrm{O}: \mathrm{C}$, 59.84; $\mathrm{H}$, $3.84 ; \mathrm{N}, 8.21$. Found: C, 59.67; H, 3.79; N, 8.13\%.

5-(1H-Indol-3-yl)-3-(4-nitrophenyl)-4,5-dihydroisoxazoline (3n). Yield: $80.62 \%$; light red solid; reaction time: 34 minutes; m.p.: $194-197^{\circ} \mathrm{C}$; IR (KBr): $\mathrm{cm}^{-1} 3217(\mathrm{~N}-\mathrm{H}), 1623(\mathrm{C}=\mathrm{C})$, $1568(\mathrm{C}=\mathrm{N}) ;{ }^{1} \mathrm{HNMR}\left(400 \mathrm{MHz} \mathrm{CDCl}_{3}, \mathrm{ppm}\right) 6.03-6.06$ (dd, 1H), 3.52-3.59 (dd, 1H), 3.67-3.74 (dd, 1H), 6.08-7.68 (m, 9H), 8.150 (brs, $1 \mathrm{H}) .{ }^{13} \mathrm{C} \mathrm{NMR}\left(100 \mathrm{MHz} \mathrm{CDCl}_{3}, \mathrm{ppm}\right)$ : 40.6, 78, 155.5, 128.4, 127.9, 129, 136.9, 115.3, 125.2, 136, $111.6,122.7,122.5,119.3,115.3$, MS: $m / z$ with +ve mode: $308.1\left(\mathrm{M}^{+\cdot}\right)$, Anal. Calcd. for $\mathrm{C}_{17} \mathrm{H}_{13} \mathrm{~N}_{3} \mathrm{O}_{3}$ : C, 66.44; $\mathrm{H}, 4.26$; $\mathrm{N}, 13.67$. Found: $\mathrm{C}, 66.47 ; \mathrm{H}, 4.41 ; \mathrm{N}, 13.54 \%$.

4.2. Antidepressant Activity. The Porsolt forced swimming test (behavioral despair test) was employed. Adult male albino Swiss-Webster $(20 \pm 2 \mathrm{~g})$ mice were used with free access to food and water. They were housed in a group of six. The approval of the Institutional Animal Ethical Committee (IAEC) of R. C. Patel Institute of Pharmaceutical Education and Research, Shirpur (Maharashtra, India) was taken prior to the start of experiments. All the synthesized compounds (3a-3n) were screened for antidepressant activity using a Porsolt forced swimming (behavioral despair) test [27]. Acute oral toxicity of synthesized compounds was determined by OECD guideline no. 425 (up and down procedure). $\mathrm{LD}_{50}$ was calculated as per OECD guidelines 425 [28].

The synthesized compounds $(100 \mathrm{mg} / \mathrm{kg})$, imipramine $(20 \mathrm{mg} / \mathrm{kg})$, and fluoxetine $(20 \mathrm{mg} / \mathrm{kg})$ suspended in aqueous tween $80(0.5 \%)$, were injected as intraperitonealy (ip) $(n=$ $6)$. After $1 / 2 \mathrm{hr}$, the mouse was dropped into the glass cylindrical container (diameter $10 \mathrm{~cm}$, height $25 \mathrm{~cm}$ ), containing approximately $20 \mathrm{~cm}$ of water at $25 \pm 1{ }^{\circ} \mathrm{C}$ temperature. Water was replaced between every trial. Each mouse left for 6 minute at the end of the first $2 \mathrm{~min}$; the animals showing initial vigorous struggling were immobile. The immobility times of each mouse were measured over the period of $4 \mathrm{~min}$. Each mouse was judged immobile when it ceased struggling and remained floating motionless in the water, making only those movements necessary to keep its head above water. Conventional antidepressants decreased the immobility time in this test. Statistical analysis was performed by one-way ANOVA followed by Dunnett's test to evaluate the results. Percentage decrease in immobility duration (\%DID) for test and standard drugs was calculated using following formula:

$$
\% \mathrm{DID}=\left[\frac{A-B}{A}\right] * 100
$$

where $A$ is the duration of immobility $(s)$ in control group and $B$ is the duration of immobility $(s)$ in test group. All the results of the antidepressant activity are given in (Table 2).

4.3. Locomotor Activity. Most of drugs acting on CNS influence the locomotor activities in man and animals. In other words, locomotor activity can be index of wakefulness (alertness) of mental activity [29]. Hence, locomotor activities of animals treated with synthesized compounds were measured using actophotometer (Spacelab, India) to examine the possible effect on locomotor activity. In brief, test animals were placed individually, in square arena of actophotometer for ten minutes, and locomotor activity was recorded (digitally displayed). The arena was cleaned with dilute alcohol and dried between trials [30]. All the results of the locomotor activity are given in Table 2 .

\section{Acknowledgments}

The authors are thankful to Dr. S. J. Surana, Principal of the institute that provided research facilities to carry out the work. The authors gratefully acknowledge SAIF, IIT, Powai, and STIC, Cochin University, Cochin, for spectral data. 


\section{References}

[1] L. E. Schechter, R. H. Ring, C. E. Beyer et al., "Innovative approaches for the development of antidepressant drugs: current and future strategies," Neuro Therapeutics, vol. 2, no. 4, pp. 590-611, 2005.

[2] P. V. Tran, F. P. Bymaster, R. K. McNamara, and W. Z. Potter, "Dual monoamine modulation for improved treatment of major depressive disorder," Journal of Clinical Psychopharmacology, vol. 23, no. 1, pp. 78-86, 2003.

[3] J. S. Biradar, B. S. Sasidhar, and R. Parveen, "Synthesis, antioxidant and DNA cleavage activities of novel indole derivatives," European Journal of Medicinal Chemistry, vol. 45, no. 9, pp. 4074-4078, 2010.

[4] E. A. RadwanRagab, N. M. Sabry, and S. M. Shenawy, "Synthesis and biological evaluation of new 3-substituted indole derivatives as potential anti-inflammatory and analgesic agents," Bioorganic and Medicinal Chemistry, vol. 15, no. 11, pp. 3832-3841, 2007.

[5] S. S. Panda and P. V. R. Chaudhari, "Synthesis of novel indolylpyrimidine antiinflammatory, antioxidant and antibacterial agents," Indian Journal of Pharmaceutical Sciences, vol. 70, no. 2, pp. 208-215, 2008.

[6] M. Amir, S. A. Javed, and H. Kumar, "Design and synthesis of 3[3-(substituted phenyl)-4-piperidin-1-ylmethyl/-4-morpholin4-ylmethyl-4,5-dihydro-isoxazol-5-yl]-1H-indoles as potent anti-inflammatory agents "' Medicinal Chemistry Research, vol. 19, no. 3, pp. 299-310, 2010.

[7] R. Patil and J. S. Biradar, "Synthesis and antimicrobial activity of 3-substituted phenyl-5-( $5^{\prime}$-substituted- $2^{\prime}$-phenylindol$3^{\prime}$-yl)-1,2-diazoles and 3-substituted phenyl-5-(5' -substituted$2^{\prime}$-phenylindol-3' -yl)-isoxazoles," Asian Journal of Chemistry, vol. 11, no. 4, pp. 1127-1136, 1999.

[8] M. Imran and S. A. Khan, "Synthesis, spectral characterization and antimicrobial activity of new isoxazoline derivatives," Oriental Journal of Chemistry, vol. 19, pp. 695-697, 2003.

[9] A. Kamal, J. Surendranadha Reddy, M. Janaki Ramaiah et al., "Design, synthesis and biological evaluation of 3,5-diarylisoxazoline/isoxazole-pyrrolobenzodiazepine conjugates as potential anticancer agents," European Journal of Medicinal Chemistry, vol. 45, no. 9, pp. 3924-3937, 2010.

[10] S. Balachandran, P. K. Gadekar, S. Parkale et al., "Synthesis and biological activity of novel MIF antagonists," Bioorganic and Medicinal Chemistry Letters, vol. 21, no. 5, pp. 1508-1511, 2011.

[11] A. Padmaja, T. Payani, G. D. Reddy, and V. Padmavathi, "Synthesis, antimicrobial and antioxidant activities of substituted pyrazoles, isoxazoles, pyrimidine and thioxopyrimidine derivatives," European Journal of Medicinal Chemistry, vol. 44, no. 11, pp. 4557-4566, 2009.

[12] G. Ahmad, P. K. Mishra, P. Gupta et al., "Synthesis of novel benzofuran isoxazolines as protein tyrosine phosphatase $1 \mathrm{~B}$ inhibitors," Bioorganic and Medicinal Chemistry Letters, vol. 16, no. 8, pp. 2139-2143, 2006.

[13] K. Traynor, "New drug and biological product approvals, 2011," American Journal of Health-System Pharmacy, vol. 69, pp. 180-182, 2012.

[14] N. Ergenec, N. S. Gunay, and S. Damirdamar, "Synthesis and antidepressant evaluation of new 3-phenyl-5-sulfonamidoindole derivatives," European Journal of Medicinal Chemistry, vol. 33, no. 2, pp. 143-148, 1998.
[15] S. M. Shelke and S. H. Bhosale, "Synthesis, antidepressant evaluation and QSAR studies of novel 2-(5H-[1,2, 4] triazino[5,6-b]indol-3-ylthio)- $N$-(substituted phenyl)acetamides," Bioorganic and Medicinal Chemistry Letters, vol. 20, no. 15, pp. 4661-4664, 2010.

[16] A. Varvaresou, T. Siatra-Papastaikoudi, A. Tsotinis, A. TsantiliKakoulidou, and A. Vamvakides, "Synthesis, lipophilicity and biological evaluation of indole-containing derivatives of 1,3,4thiadiazole and 1,2,4-triazole," Farmaco, vol. 53, no. 5, pp. 320-326, 1998.

[17] Y. R. Prasad, P. R. Kumar, and B. Ramesh, International Journal of Chemical Sciences, vol. 5, no. 2, pp. 542-548, 2007.

[18] H. S. Youn, E. J. Lee, J. E. Lee et al., "Synthesis and biological evaluation of isoxazoline and isoxazole derivatives as 5-HT2A and 5-HT2C receptor ligands," Bulletin of the Korean Chemical Society, vol. 30, no. 8, pp. 1873-1876, 2009.

[19] V. Tiwari, A. Parvez, and J. Meshram, "Benign methodology and improved synthesis of 5-(2-chloroquinolin-3-yl)-3- phenyl-4,5dihydroisoxazoline using acetic acid aqueous solution under ultrasound irradiation," Ultrasonics Sonochemistry, vol. 18, no. 5, pp. 911-916, 2011.

[20] S. S. Panda, P. V. R. Chowdary, and B. S. Jayashree, "Synthesis, antiinflammatory and antibacterial activity of novel indolylisoxazoles," Indian Journal of Pharmaceutical Sciences, vol. 71, no. 6, pp. 684-687, 2009.

[21] M. Kidwai, S. Kukreja, and R. Thakur, "K2CO3-mediated regioselective synthesis of isoxazoles and pyrazolines," Letters in Organic Chemistry, vol. 3, no. 2, pp. 135-139, 2006.

[22] P. Lidström, J. Tierney, B. Wathey, and J. Westman, "Microwave assisted organic synthesis-a review," Tetrahedron, vol. 57, no. 45, pp. 9225-9283, 2001.

[23] S. Caddick, "Microwave assisted organic reactions," Tetrahedron, vol. 51, no. 38, pp. 10403-10432, 1995.

[24] N. Philip James and H. R. Snyder, "INDOLE-3-ALDEHYDE," Organic Syntheses, vol. 4, p. 539, 1963.

[25] M. Zahran and A. M. Ibrahm, "Synthesis and cellular cytotoxicities of new $N$-substituted indole-3-carbaldehyde and their indolylchalcones "' Journal of Chemical Sciences, vol. 121, no. 4, pp. 455-462, 2009.

[26] X. H. Guo, X. X. Qi, and J. B. Chang, "5-(1H-indol-3-yl)-3-(4methylphenyl)-4,5-dihydroisoxazoline," Acta Crystallographica Section E, vol. 62, no. 1, pp. o374-o375, 2006.

[27] R. D. Porsolt, M. Le Pichon, and M. Jalfre, "Depression: a new animal model sensitive to antidepressant treatments," Nature, vol. 266, no. 5604, pp. 730-732, 1977.

[28] OECD, 2001-gudeline on acute oral toxicity (AOT) Environmental health and safety monograph series on testing and adjustment No. 425.

[29] S. K. Kulkarni, Hand Book of Experimental Pharmacology, Vallabh Publications, Delhi, India, 3rd edition, 2007.

[30] R. P. Mahesh, B. K. Sharma, S. B. Gautam, and D. K. Pandey, International Journal of Pharma and Bio Sciences, vol. 1, pp. 1-6, 2010. 

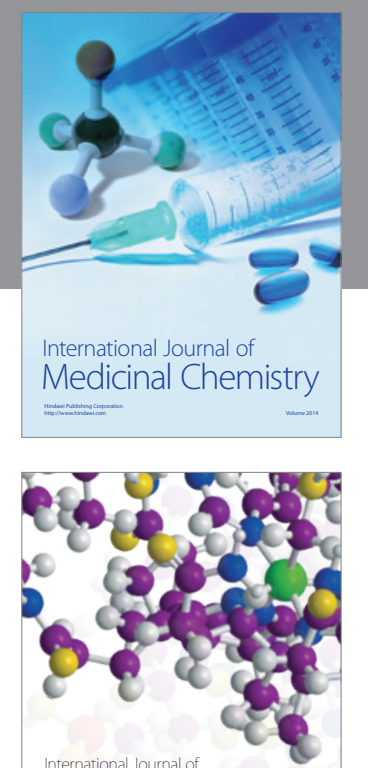

\section{Carbohydrate} Chemistry

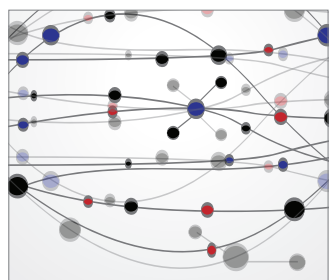

The Scientific World Journal
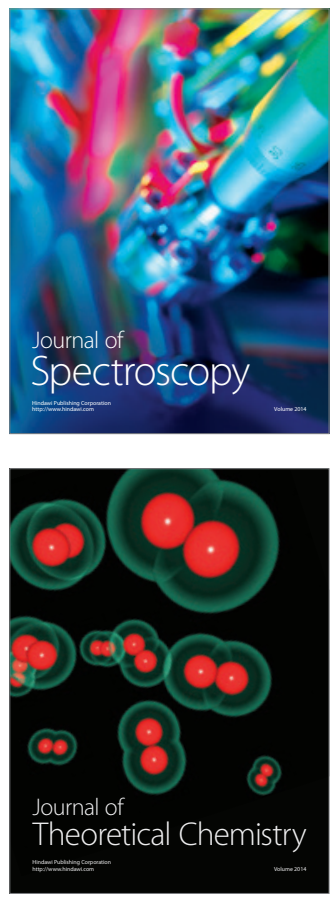
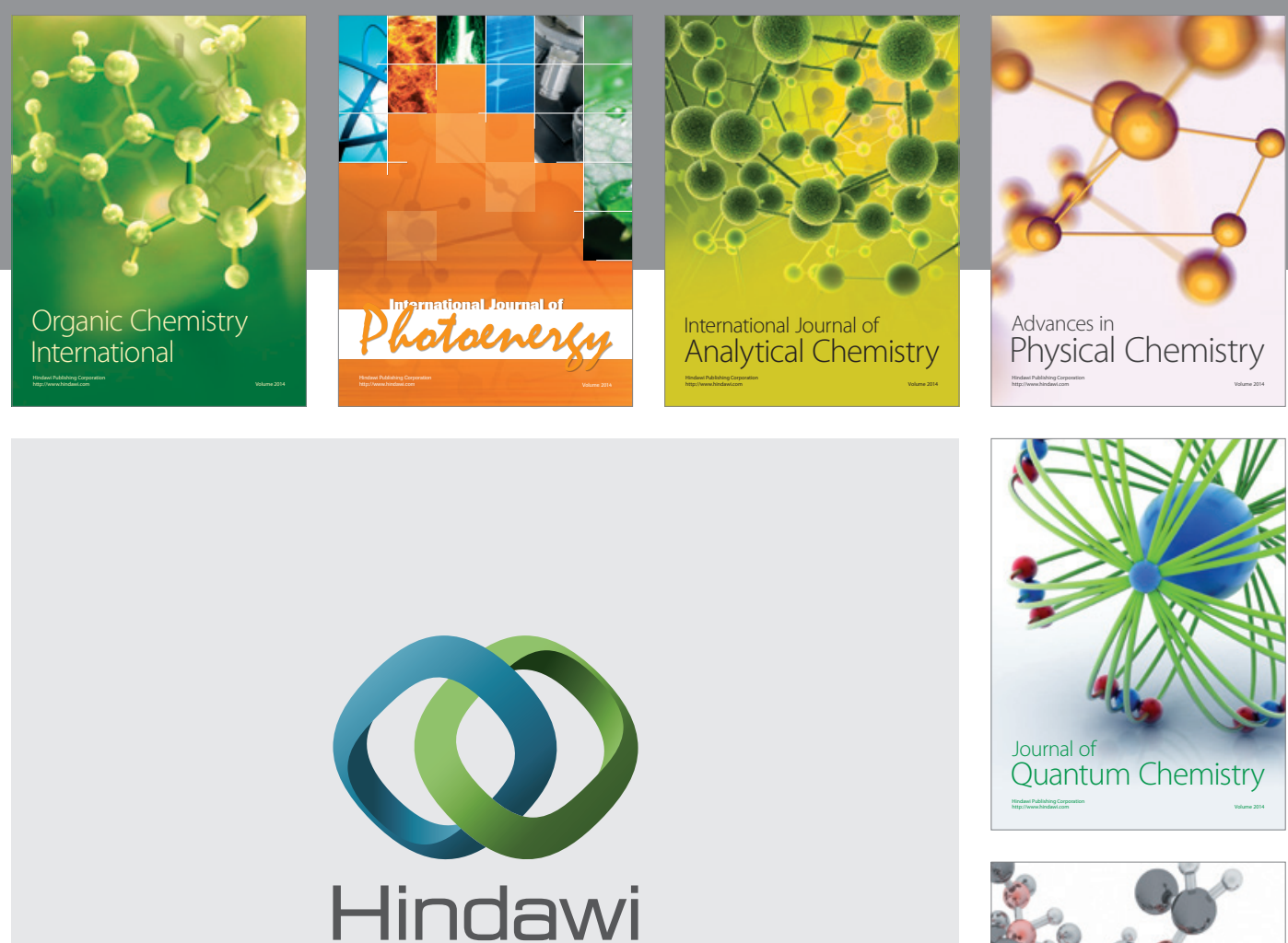

Submit your manuscripts at

http://www.hindawi.com

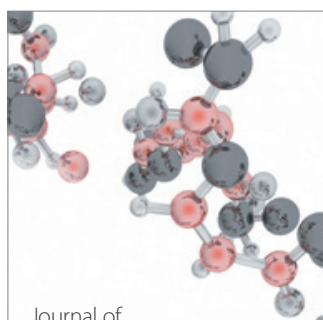

Analytical Methods

in Chemistry

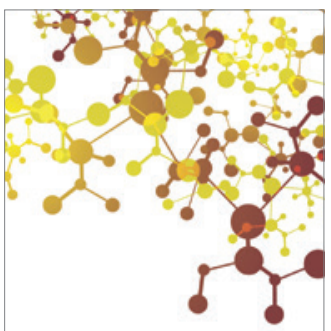

Journal of

Applied Chemistry

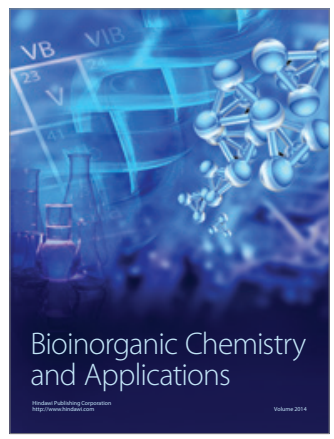

Inorganic Chemistry
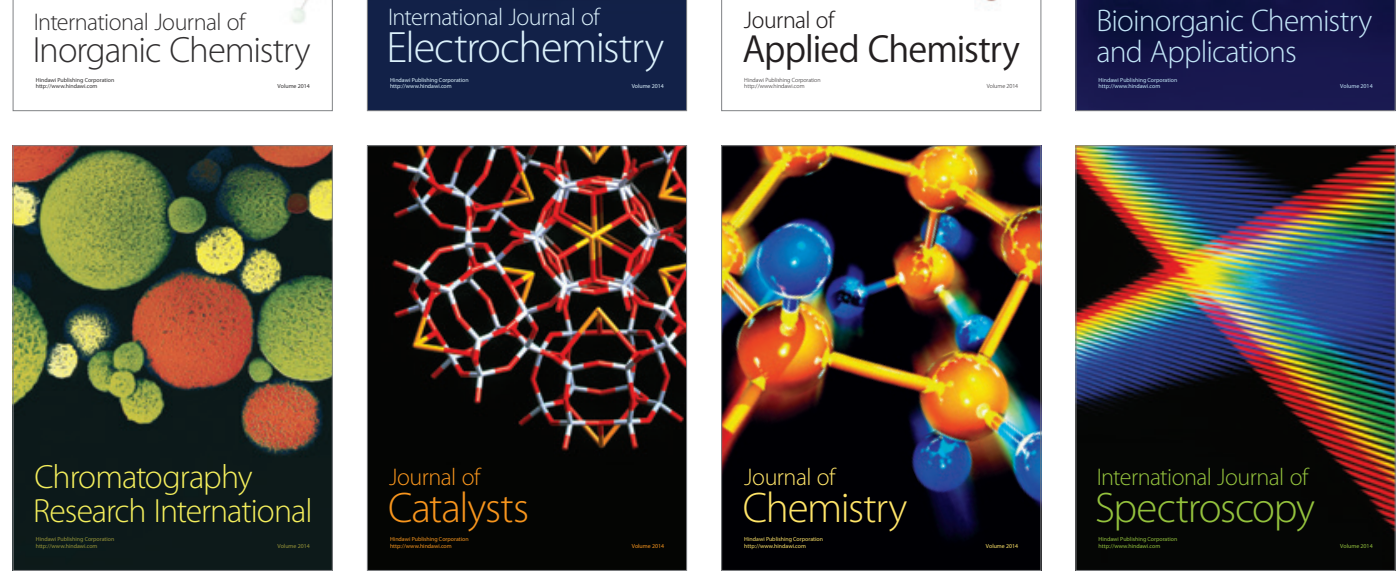